\title{
Symmetries and first integrals of ordinary difference equations
}

\author{
BY P. E. HYDON \\ Department of Mathematics and Statistics, University of Surrey, \\ Guildford GU2 7XH, UK (P.Hydon@surrey.ac.uk)
}

Received 3 December 1999; revised 31 May 2000; accepted 13 June 2000

This paper describes a new symmetry-based approach to solving a given ordinary difference equation. By studying the local structure of the set of solutions, we derive a systematic method for determining one-parameter Lie groups of symmetries in closed form. Such groups can be used to achieve successive reductions of order. If there are enough symmetries, the difference equation can be completely solved. Several examples are used to illustrate the technique for transitive and intransitive symmetry groups. It is also shown that every linear second-order ordinary difference equation has a Lie algebra of symmetry generators that is isomorphic to $\mathfrak{I}((3)$. The paper concludes with a systematic method for constructing first integrals directly, which can be used even if no symmetries are known.

Keywords: difference equations; symmetry analysis; Lie groups; first integrals

\section{Introduction}

Over a century ago, Sophus Lie introduced symmetry-based techniques for solving ordinary differential equations (ODEs). Lie's approach enables the user to determine Lie groups of symmetries of a given ODE. If a sufficiently large symmetry group can be found, it may be used to solve the ODE. For an introduction to symmetry methods for ODEs, see Olver (1993), Bluman \& Kumei (1989), Stephani (1989) or Hydon (2000).

Maeda (1987) has shown that autonomous systems of first-order ordinary difference equations $(\mathrm{O} \Delta \mathrm{Es})$ can be simplified or solved using an extension of Lie's method. Maeda also showed that the linearized symmetry condition (LSC) for such O $\Delta \mathrm{Es}$ amounts to a set of functional equations. In general, these are hard to solve, but Maeda described two examples for which a very restrictive ansatz yields Lie symmetries. Gaeta (1993) uses formal series expansions to derive some symmetries of those systems of $\mathrm{O} \Delta \mathrm{Es}$ that are discretizations of continuous systems. Given an ODE with known Lie point symmetries, one may ask whether it is possible to discretize the ODE in a way that preserves at least some of the symmetries. Dorodnitsyn (1994) describes how this can be achieved, and lists some classes of O $\Delta$ Es that have a given Lie group. Dorodnitsyn et al. (2000) uses the same technique to discretize the second-order ODEs that arise from the classification of Lie groups acting on the real plane.

Maeda's ideas have been extended to non-autonomous systems and higher-order O $\Delta$ Es by Quispel \& Sahadevan (1993) and Levi et al. (1997). These papers describe 
different series-based methods for obtaining some solutions of the LSC. Series expansions can be calculated if the symmetry condition has a fixed point, although it is usually not obvious how to sum the series to obtain solutions in closed form. Unfortunately, the well-known method for calculating invariants requires the symmetry generator to be in closed form. This is a substantial limitation on the usefulness of series-based techniques.

In the current paper, we introduce a systematic method for obtaining Lie symmetries (in closed form) of a given $\mathrm{O} \Delta \mathrm{E}$. For ODEs, the most general class of local symmetries is the class of dynamical (or internal) symmetries (see Stephani 1989; Anderson et al. 1993). This class includes the trivial symmetries, which map every solution of the ODE to itself and cannot be used to solve the ODE. It is usual to factor out the trivial symmetries by restricting attention to those dynamical symmetries that leave the independent variable unchanged (which are called vertical or evolutionary symmetries). Two symmetries are said to be equivalent if they differ by a trivial symmetry, so every local symmetry of an ODE is equivalent to a vertical symmetry. In $\S 2$ we study the action of symmetries on the set of solutions of a given $\mathrm{O} \Delta \mathrm{E}$. We prove that every local symmetry is equivalent to a symmetry that leaves the independent variable unchanged. This result immediately frees us from a maze of technical complications that are associated with the fact that the independent variable takes discrete values.

Every ODE has infinitely many independent one-parameter Lie groups of vertical symmetries. However, they can only be found by looking for generators of a restricted form. For example, all one-parameter Lie groups of vertical symmetries of the secondorder ODE

$$
u^{\prime \prime}=\omega\left(x, u, u^{\prime}\right)
$$

have generators of the form

$$
X=Q\left(x, u, u^{\prime}\right) \frac{\partial}{\partial u} .
$$

(These symmetries are equivalent to contact symmetries.) The set of all generators (1.2) cannot be found until the general solution of (1.1) is known. However, the one-parameter Lie groups of point symmetries are equivalent to vertical symmetries whose generators (1.2) have $Q$ linear in $u^{\prime}$. By placing this restriction on $Q$, it is usually possible to obtain the Lie point symmetries systematically. In $\S 3$ we show, using various examples, how to use the same idea to construct Lie symmetries of $\mathrm{O} \Delta \mathrm{Es}$ (in closed form). Starting from the LSC, which is a functional equation, we derive an associated system of linear differential equations. This system is similar to the system of determining equations for Lie symmetries of a given ODE. Moreover, having set up the mathematical framework for the new method, we find that it enables us to transfer the main symmetry methods for ODEs across to O $\Delta$ Es. Some minor modifications are needed, and they are described in $\S 4$.

A recent paper by Anco \& Bluman (1998) describes a constructive method for obtaining first integrals of ODEs directly, without using Lie symmetries. Instead, the method uses the adjoint of the LSC. In $\S 5$ we introduce a similar technique for obtaining first integrals of $\mathrm{O} \Delta$ Es directly. This makes it possible to solve some $\mathrm{O} \Delta \mathrm{Es}$ for which no Lie symmetries can be found. 


\section{Symmetries of ordinary difference equations}

We begin by considering the structure of the set of solutions of the $N$ th-order $\mathrm{O} \Delta \mathrm{E}$

$$
u_{n+N}=\omega\left(n, u_{n}, u_{n+1}, \ldots, u_{n+N-1}\right), \quad \frac{\partial \omega}{\partial u_{n}} \neq 0,
$$

where $\omega$ is a given function. We restrict attention to regions in which $\omega$ is a smooth function of its arguments. The independent variable $n$ is an integer. Some authors prefer to use $x_{n}$ as the independent variable (particularly if the $\mathrm{O} \Delta \mathrm{E}$ arises as a discretization of an ODE). There is no essential difference between these formulations, provided that there is a bijection that maps $n$ to $x_{n}$. (Note that the meshpoints, $x_{n}$, need not be uniformly spaced.)

Our objective is to obtain the general solution of (2.1). If the values $u_{n_{0}+k}$, $k=0, \ldots, N-1$, are known for one particular $n_{0}$, then the $\mathrm{O} \Delta \mathrm{E}(2.1)$ determines $u_{n}$ recursively for $n \geqslant n_{0}+N$. Therefore, the general solution depends on $N$ arbitrary constants, $c^{i}$, and can be written (in principle) in the form

$$
u_{n}=F\left(n, c^{1}, \ldots, c^{N}\right) .
$$

Furthermore, because the arbitrary constants are independent, it is possible to solve the system

$$
u_{n+k}=F\left(n+k, c^{1}, \ldots, c^{N}\right), \quad k=0, \ldots, N-1,
$$

to obtain each $c^{i}$ in the form

$$
c^{i}=\phi^{i}\left(n, u_{n}, \ldots, u_{n+N-1}\right), \quad i=1, \ldots, N .
$$

The $N$ functions $\phi^{i}$ are functionally independent first integrals of the $\mathrm{O} \Delta \mathrm{E}(2.1)$. Here we use the same terminology as for ODEs: a first integral is a function that is not identically constant, but is constant on all solutions of the given $\mathrm{O} \Delta \mathrm{E}$. The term 'functionally independent' means that the Jacobian does not vanish, that is,

$$
\frac{\partial\left(\phi^{1}, \ldots, \phi^{N}\right)}{\partial\left(u_{n}, \ldots, u_{n+N-1}\right)} \neq 0 \text {. }
$$

Throughout this paper, we shall work in the space of variables $n, u_{n}, \ldots, u_{n+N-1}$; the condition (2.5) enables us to use $n, \phi^{1}, \ldots, \phi^{N}$ as an alternative set of (local) coordinates. Therefore, any function $g\left(n, u_{n}, \ldots, u_{n+N-1}\right)$ can be rewritten in the form

$$
g\left(n, u_{n}, \ldots, u_{n+N-1}\right)=G\left(n, \phi^{1}, \ldots, \phi^{N}\right)
$$

for some function $G$. In particular, every first integral $\phi\left(n, u_{n}, \ldots, u_{n+N-1}\right)$ is a function of the first integrals $\phi^{i}$ only.

It is useful to introduce the shift operator, which acts on the independent variable $n$ as follows:

$$
\mathcal{S}: n \mapsto n+1 \text {. }
$$

The action of this operator on any given function of $n$ only, $g(n)$, is defined by the action on the function's arguments:

$$
\mathcal{S}(g(n))=g(\mathcal{S}(n))=g(n+1) .
$$


By applying the shift operator to any solution (2.2) of the $\mathrm{O} \Delta \mathrm{E}$, we can use $(2.3)$ and (2.7) to obtain

$$
\mathcal{S}\left(u_{n}\right)=F\left(\mathcal{S}(n), c^{1}, \ldots, c^{N}\right)=u_{n+1},
$$

because each $c^{i}$ is independent of $n$. Similarly,

$$
\mathcal{S}\left(u_{n+k}\right)=u_{n+k+1}, \quad k=0, \ldots, N-2 .
$$

Equation $(2.8 a)$ also holds for $k=N-1$, but because $u_{n}$ is a solution of the $\mathrm{O} \Delta \mathrm{E}$, we can replace $u_{n+N}$ by the right-hand side of (2.1):

$$
\mathcal{S}\left(u_{n+N-1}\right)=\omega\left(n, u_{n}, u_{n+1}, \ldots, u_{n+N-1}\right) .
$$

From here on, we restrict our attention to solutions of the $\mathrm{O} \Delta \mathrm{E}$, and so we regard $\mathcal{S}$ as an operator on $n$ and $u_{n+k}, k=0, \ldots, N-1$, whose action is defined by (2.6), $(2.8 a)$ and $(2.8 b)$. From $(2.7)$, the action of $\mathcal{S}$ on functions of these variables is

$$
\mathcal{S}\left(g\left(n, u_{n}, \ldots, u_{n+N-2}, u_{n+N-1}\right)\right)=g\left(n+1, u_{n+1}, \ldots, u_{n+N-1}, \omega\right) .
$$

If $\phi$ is a first integral, it is constant on the solutions of the $\mathrm{O} \Delta \mathrm{E}$, and, hence,

$$
\mathcal{S} \phi=\phi \text {. }
$$

Therefore, from (2.9), the first integrals of (2.1) are the non-constant solutions of

$$
\phi\left(n+1, u_{n+1}, \ldots, \omega\left(n, u_{n}, \ldots, u_{n+N-1}\right)\right)=\phi\left(n, u_{n}, \ldots, u_{n+N-1}\right) .
$$

This condition holds as an identity in all variables. In $\S 5$ we shall use it to develop a constructive technique for obtaining first integrals.

A symmetry, $\Gamma$, of (2.1) maps the set of solutions to itself. Therefore, if

$$
\Gamma:\left(n, \phi^{1}, \ldots, \phi^{N}\right) \mapsto\left(\hat{n}, \hat{\phi}^{1}, \ldots, \hat{\phi}^{N}\right),
$$

each $\hat{\phi}^{i}$ is a function of $\phi^{1}, \ldots, \phi^{N}$ only. At this point, it is necessary to ask what class of maps $\Gamma$ respect the structure of an $\mathrm{O} \Delta \mathrm{E}$, and how these differ from the symmetries of an ODE.

Dynamical symmetries of a given $N$ th-order ODE are defined similarly to (2.12), but with the independent variable $x$ replacing $n$. It is helpful to regard the first integrals $\phi^{1}, \ldots, \phi^{N}$ as local coordinates on an $N$-dimensional fibre over each base point $x$. Dynamical symmetries of the ODE are (locally) diffeomorphisms of the trivial fibre bundle $\mathbb{R} \times \mathbb{R}^{N}$, with the property that each $\hat{\phi}^{i}$ is independent of $x$. (The base space spanned by the independent variable $x$ is locally isomorphic to $\mathbb{R}$; each fibre is locally isomorphic to $\mathbb{R}^{N}$.)

The first integrals of the $\mathrm{O} \Delta \mathrm{E}(2.1)$ also form an $N$-dimensional fibre over each base point; just as for ODEs, the fibre is locally isomorphic to $\mathbb{R}^{N}$. However, the independent variable $n$ takes only integer values, so the base points are discrete. The fibres are not connected, and symmetries of the $\mathrm{O} \Delta \mathrm{E}$ must preserve this disjoint structure. Hence, they are required to be fibre-preserving (that is, they map fibres to fibres), the consequence of which is that $\hat{n}$ is a function of $n$ only. Moreover, the set of base points has the natural ordering inherited from $\mathbb{Z}$, and so the action of any symmetry on the base points should be consistent with this ordering. In other words, 
fibres must not be shuffled; symmetries are required to be neighbour-preserving. This can happens in one of two ways: either a symmetry is order-preserving, in which case

$$
\hat{n}(n+1)=\hat{n}(n)+1,
$$

or else the symmetry is order-reversing, in which case its action on $n$ is equivalent to a reflection. Discrete symmetries are not investigated in this paper, so we need only consider the order-preserving symmetries (which include all one-parameter Lie groups of symmetries). Just as for ODEs, the functions $\hat{\phi}^{i}\left(\phi^{1}, \ldots, \phi^{N}\right)$ in $(2.12)$ are required to be diffeomorphisms (locally at least).

A symmetry is trivial if every solution is mapped to itself, that is, if

$$
\hat{\phi}^{i}=\phi^{i}, \quad i=1, \ldots, N .
$$

Lemma 2.1. For each $k \in \mathbb{Z}$, the transformation generated by $\mathcal{S}^{k}$ is a trivial symmetry of (2.1). (Note: if $k$ is negative, $\mathcal{S}^{k}$ denotes $\left(\mathcal{S}^{-1}\right)^{-k}$.)

Proof. For $k \geqslant 0$, apply $\mathcal{S}$ repeatedly to obtain

$$
\mathcal{S}^{k}:\left(n, \phi^{1}, \ldots, \phi^{N}\right) \mapsto\left(n+k, \phi^{1}, \ldots, \phi^{N}\right) .
$$

Hence, every solution $\phi^{i}=c^{i}$ is mapped to itself. Equation (2.14) also holds for $k<0$, because (2.10) implies that $\mathcal{S}^{-1} \phi^{i}=\phi^{i}$. The action of the operator $\mathcal{S}^{-1}$ on $u_{n}$ is obtained by first using (2.1) to write $u_{n}$ as a function of $n, u_{n+1}, \ldots, u_{n+N}$, then replacing $n$ by $n-1$; the condition $\partial \omega / \partial u_{n} \neq 0$ ensures that this is possible.

One consequence of lemma 2.1 is that every non-trivial order-preserving symmetry can be regarded as the composition of a vertical (or evolutionary) symmetry, which acts only on the first integrals $\phi^{i}$ (leaving $n$ unchanged), and a trivial symmetry. Just as for ODEs, it is only the non-trivial symmetries that can be used to solve $\mathrm{O} \Delta \mathrm{Es}$, so we lose nothing by concentrating on vertical symmetries.

Lemma 2.2. Every order-preserving symmetry (2.12) is equivalent to a vertical symmetry,

$$
\tilde{\Gamma}:\left(n, \phi^{1}, \ldots, \phi^{N}\right) \mapsto\left(n, \tilde{\phi}^{1}, \ldots, \tilde{\phi}^{N}\right)
$$

Proof. The proof is by construction:

$$
\tilde{\Gamma}=\mathcal{S}^{n-\hat{n}(n)} \Gamma
$$

is the unique vertical symmetry that is equivalent to $\Gamma$. (The condition that $\Gamma$ is order-preserving ensures that $n-\hat{n}(n)$ is independent of $n$.)

In view of lemma 2.2 , we shall consider only vertical symmetries from now on. Accordingly we seek symmetries (2.12) with $\hat{n}=n$. In terms of the original variables,

$$
\Gamma:\left(n, u_{n}, \ldots, u_{n+N-1}\right) \mapsto\left(n, \hat{u}_{n}, \ldots, \hat{u}_{n+N-1}\right) .
$$

The action of $\Gamma$ on the variables $u_{n+k}$ is determined by the action on $u_{n}$. To see this, suppose that

$$
\hat{u}_{n}=g\left(n, u_{n}, \ldots, u_{n+N-1}\right)=G\left(n, \phi^{1}, \ldots, \phi^{N}\right) .
$$


Then, on the set of solutions of the $\mathrm{O} \Delta \mathrm{E}(2.1)$,

$$
\hat{u}_{n+k}=G\left(n+k, \phi^{1}, \ldots, \phi^{N}\right)=\mathcal{S}^{k} \hat{u}_{n}, \quad k=1, \ldots, N .
$$

Conditions (2.16) are analogous to the prolongation formulae for dynamical symmetries of ODEs, which reflect the necessity for contact conditions to be satisfied on the set of solutions.

The symmetry condition for the $\mathrm{O} \Delta \mathrm{E}(2.1)$ is

$$
\hat{u}_{n+N}=\omega\left(n, \hat{u}_{n}, \ldots, \hat{u}_{n+N-1}\right), \quad \text { when }(2.1) \text { holds. }
$$

Lie symmetries are obtained by linearizing the symmetry condition about the identity, as follows. We seek one-parameter (local) Lie groups of symmetries of the form

$$
\hat{u}_{n}=u_{n}+\epsilon Q\left(n, u_{n}, \ldots, u_{n+N-1}\right)+O\left(\epsilon^{2}\right) .
$$

The function $Q$ is called the characteristic of the one-parameter group. From the prolongation formulae (2.16), we obtain

$$
\hat{u}_{n+k}=u_{n+k}+\epsilon \mathcal{S}^{k} Q+O\left(\epsilon^{2}\right), \quad k=1, \ldots, N .
$$

Expanding (2.17) to first order in $\epsilon$ yields the LSC

$$
\mathcal{S}^{N} Q-X \omega=0
$$

where

$$
X=Q \frac{\partial}{\partial u_{n}}+(\mathcal{S} Q) \frac{\partial}{\partial u_{n+1}}+\cdots+\left(\mathcal{S}^{N-1} Q\right) \frac{\partial}{\partial u_{n+N-1}} .
$$

Note that when the symmetry generator $X$ is written in terms of the first integrals it has the form

$$
X=F^{1}\left(\phi^{1}, \ldots, \phi^{N}\right) \frac{\partial}{\partial \phi^{1}}+\cdots+F^{N}\left(\phi^{1}, \ldots, \phi^{N}\right) \frac{\partial}{\partial \phi^{N}},
$$

because each $\hat{\phi}^{i}$ is a function of $\Phi=\left(\phi^{1}, \ldots, \phi^{N}\right)$ only. From (2.20), we can deduce that $X$ and $\mathcal{S}$ commute as operators on functions. Given any sufficiently smooth function,

$$
g\left(n, u_{n}, \ldots, u_{n+N-1}\right)=G(n, \Phi)
$$

(2.20) implies that

$$
\mathcal{S}(X G)=\mathcal{S}\left(F^{i}(\Phi) \frac{\partial G}{\partial \phi^{i}}(n, \Phi)\right)=F^{i}(\Phi) \frac{\partial G}{\partial \phi^{i}}(n+1, \Phi)=X(\mathcal{S} G) .
$$

Therefore,

$$
\mathcal{S}(X g)=X(\mathcal{S} g)
$$

In $\S 4$, we use $(2.21)$ to derive symmetry reductions of $\mathrm{O} \Delta \mathrm{Es}$.

Just as for ODEs, the LSC is both necessary and sufficient to obtain the local Lie group of symmetries generated by $X$. To find solutions of (2.18), we must impose some constraint upon $Q$, in order to be able to split (2.18) into an overdetermined system of equations. For example, if we seek characteristics that are independent of $u_{n+N-1}$, it may be possible to split the LSC by equating powers of $u_{n+N-1}$. Before this can be achieved, some work is needed to transform (2.18) from a functional equation into a differential equation for $Q$. The next section introduces a method for accomplishing this transformation. 


\section{How to construct the determining equations}

Before describing the method for determining Lie symmetries of a general $\mathrm{O} \Delta \mathrm{E}$, we examine its main features in the context of a fairly simple example. Consider the $\mathrm{O} \Delta \mathrm{E}$

$$
u_{n+2}=\frac{u_{n} u_{n+1}}{2 u_{n}-u_{n+1}} .
$$

As this is a second-order equation, let us seek point symmetries, whose characteristics are of the form $Q=Q\left(n, u_{n}\right)$. The LSC (2.18) is

$$
Q(n+2, \omega)-\frac{2 u_{n}^{2}}{\left(2 u_{n}-u_{n+1}\right)^{2}} Q\left(n+1, u_{n+1}\right)+\frac{u_{n+1}^{2}}{\left(2 u_{n}-u_{n+1}\right)^{2}} Q\left(n, u_{n}\right)=0,
$$

where $\omega$ denotes the right-hand side of (3.1). The chief difficulty with (3.2) is that the function $Q$ takes three separate pairs of arguments. To overcome this difficulty, we differentiate (3.2) with respect to $u_{n}$, keeping $\omega$ fixed. Here $u_{n+1}$ is regarded as a function of $n, u_{n}$, and $\omega$. A standard result from multivariable calculus yields

$$
\frac{\partial u_{n+1}\left(n, u_{n}, \omega\right)}{\partial u_{n}}=-\frac{\partial \omega / \partial u_{n}}{\partial \omega / \partial u_{n+1}}=\frac{u_{n+1}^{2}}{2 u_{n}^{2}} .
$$

Therefore, we apply the differential operator

$$
\frac{\partial}{\partial u_{n}}+\frac{u_{n+1}^{2}}{2 u_{n}^{2}} \frac{\partial}{\partial u_{n+1}}
$$

to (3.2), and obtain

$$
\begin{aligned}
& -\frac{u_{n+1}^{2}}{\left(2 u_{n}-u_{n+1}\right)^{2}} Q^{\prime}\left(n+1, u_{n+1}\right)+\frac{2 u_{n+1}}{\left(2 u_{n}-u_{n+1}\right)^{2}} Q\left(n+1, u_{n+1}\right) \\
& +\frac{u_{n+1}^{2}}{\left(2 u_{n}-u_{n+1}\right)^{2}} Q^{\prime}\left(n, u_{n}\right)-\frac{2 u_{n+1}^{2}}{u_{n}\left(2 u_{n}-u_{n+1}\right)^{2}} Q\left(n, u_{n}\right)=0,
\end{aligned}
$$

where a prime denotes a derivative with respect to the continuous variable. This simplifies to

$$
-Q^{\prime}\left(n+1, u_{n+1}\right)+\frac{2}{u_{n+1}} Q\left(n+1, u_{n+1}\right)+Q^{\prime}\left(n, u_{n}\right)-\frac{2}{u_{n}} Q\left(n, u_{n}\right)=0 .
$$

Now differentiate (3.3) with respect to $u_{n}$, this time keeping $u_{n+1}$ fixed, to obtain the ODE

$$
\frac{\mathrm{d}}{\mathrm{d} u_{n}}\left(Q^{\prime}\left(n, u_{n}\right)-\frac{2}{u_{n}} Q\left(n, u_{n}\right)\right)=0 .
$$

Note that $n$ appears only as a parameter at this stage. The general solution of (3.4) is

$$
Q\left(n, u_{n}\right)=A(n) u_{n}+B(n) u_{n}^{2} .
$$

Substituting (3.5) into (3.3) yields the $\mathrm{O} \Delta \mathrm{E}$

$$
A(n+1)=A(n),
$$


whose general solution is

$$
A(n)=c^{1} \text {. }
$$

The remaining unknown function, $B(n)$, is determined by substituting (3.5) into the original LSC (3.2). This leads to the simple linear $\mathrm{O} \Delta \mathrm{E}$

$$
B(n+2)-2 B(n+1)+B(n)=0,
$$

whose general solution is

$$
B(n)=c^{2} n+c^{3} .
$$

Summarizing these results, we have found a three-dimensional Lie algebra of symmetry generators, whose characteristics are linear combinations of

$$
Q_{1}=u_{n}, \quad Q_{2}=n u_{n}^{2}, \quad Q_{3}=u_{n}^{2} .
$$

The method used above exploits the fact that each of the unknown functions in the LSC is invariant under a first-order differential operator. The same method can be used to tackle any $\mathrm{O} \Delta \mathrm{E}(2.1)$, although the complexity of the calculations increases with $N$. For simplicity, we focus mainly on second-order O $\Delta$ Es:

$$
u_{n+2}=\omega\left(n, u_{n}, u_{n+1}\right), \quad \frac{\partial \omega}{\partial u_{n}} \neq 0, \quad \frac{\partial \omega}{\partial u_{n+1}} \neq 0 .
$$

(Note: the condition $\partial \omega / \partial u_{n+1} \neq 0$ ensures that the $\mathrm{O} \Delta \mathrm{E}$ is genuinely second order, not equivalent to a first-order problem with step length 2.) The LSC for point symmetries is

$$
Q(n+2, \omega)-\frac{\partial \omega}{\partial u_{n+1}} Q\left(n+1, u_{n+1}\right)-\frac{\partial \omega}{\partial u_{n}} Q\left(n, u_{n}\right)=0 .
$$

By eliminating $Q(n+2, \omega)$ and $Q\left(n+1, u_{n+1}\right)$, we can use (3.8) to derive an ODE for $Q\left(n, u_{n}\right)$ that is of order three or less. First, differentiate (3.8) with respect to $u_{n}$, keeping $\omega$ fixed, to obtain (after simplification)

$$
Q^{\prime}\left(n+1, u_{n+1}\right)+\frac{\partial \lambda}{\partial u_{n+1}} Q\left(n+1, u_{n+1}\right)-Q^{\prime}\left(n, u_{n}\right)+\frac{\partial \lambda}{\partial u_{n}} Q\left(n, u_{n}\right)=0,
$$

where

$$
\lambda=\ln \left|\frac{\partial \omega}{\partial u_{n+1}}\right|-\ln \left|\frac{\partial \omega}{\partial u_{n}}\right|
$$

Differentiating $(3.9)$ with respect to $u_{n}$, keeping $u_{n+1}$ fixed, we obtain

$$
\frac{\partial^{2} \lambda}{\partial u_{n} \partial u_{n+1}} Q\left(n+1, u_{n+1}\right)-Q^{\prime \prime}\left(n, u_{n}\right)+\frac{\partial \lambda}{\partial u_{n}} Q^{\prime}\left(n, u_{n}\right)+\frac{\partial^{2} \lambda}{\partial u_{n} \partial u_{n}} Q\left(n, u_{n}\right)=0 .
$$

If $\partial^{2} \lambda / \partial u_{n} \partial u_{n+1}=0$, equation (3.10) is a second-order ODE for $Q\left(n, u_{n}\right)$. Otherwise, we must divide (3.10) by $\partial^{2} \lambda / \partial u_{n} \partial u_{n+1}$ and differentiate once more with respect to $u_{n}$ (keeping $u_{n+1}$ fixed) to obtain a third-order ODE for $Q\left(n, u_{n}\right)$. Typically, the coefficients in the reduced ODE depend upon $u_{n+1}$. If this occurs, the ODE can be split by gathering together all terms with the same dependence upon $u_{n+1}$. 
The solution of the reduced ODE contains arbitrary functions of $n$. It is substituted into the LSC, which can then be split into a system of determining O $\Delta$ Es for the arbitrary functions (by grouping together all terms with the same dependence upon $u_{n}$ and $\left.u_{n+1}\right)$. These determining equations are usually very easy to solve.

So far, we have imposed the restriction that $Q$ is independent of $u_{n+1}$. This keeps the calculations fairly simple, but many $\mathrm{O} \Delta \mathrm{Es}$ have no symmetries of this form. Lie point symmetries of ODEs have a characteristic $Q$ that is linear in the first derivative of the dependent variable. This suggests that a similar ansatz may be useful for $\mathrm{O} \Delta \mathrm{Es}$. If

$$
Q=a\left(n, u_{n}\right) u_{n+1}+b\left(n, u_{n}\right)
$$

for some functions $a, b$, the LSC (2.18) amounts to

$$
\begin{aligned}
a(n+2, \omega) \mathcal{S} \omega+b(n+2, \omega)-\frac{\partial \omega}{\partial u_{n+1}} & \left\{a\left(n+1, u_{n+1}\right) \omega+b\left(n+1, u_{n+1}\right)\right\} \\
& -\frac{\partial \omega}{\partial u_{n}}\left\{a\left(n, u_{n}\right) u_{n+1}+b\left(n, u_{n}\right)\right\}=0 .
\end{aligned}
$$

This is reduced to a set of ODEs for $a\left(n, u_{n}\right)$ and $b\left(n, u_{n}\right)$ in essentially the same way as before. First differentiate with respect to $u_{n}$, keeping $\omega$ fixed, to eliminate $b(n+2, \omega)$. Then multiply the result by whatever factor is needed to obtain an equation of the form

$$
a(n+2, \omega)+\text { other terms }=0 .
$$

Differentiate this with respect to $u_{n}$, keeping $\omega$ fixed, to eliminate $a(n+2, \omega)$. Next, eliminate all terms containing $a\left(n+1, u_{n+1}\right), b\left(n+1, u_{n+1}\right)$, and their derivatives. This is achieved by first multiplying the equation by whatever factor is needed to isolate one of these functions, then differentiating with respect to $u_{n}$ (keeping $u_{n+1}$ fixed). The process is repeated until all undesirable terms have been removed. Finally, the resulting ODE is split into a set of ODEs (by equating terms with the same dependence upon $u_{n+1}$ ). This approach readily generalizes to any other ansatz for $Q$ and to higher-order $\mathrm{O} \Delta$ Es. The calculations rapidly become too lengthy to be done by hand, but can be done with the aid of computer algebra. For the remainder of this paper, we shall state symmetries without describing the details of their derivation.

The method described above is capable of yielding more symmetries than have been found previously by fixed-point summations. For example, Quispel \& Sahadevan (1993) used a fixed-point method to look for symmetries of

$$
u_{n+2}=\frac{2 u_{n+1}-u_{n}\left(1-u_{n+1}^{2}\right)}{1-u_{n+1}^{2}+2 u_{n} u_{n+1}} .
$$

They found two independent characteristics of the form $Q=Q\left(n, u_{n}\right)$, namely

$$
Q_{1}=u_{n}^{2}+1, \quad Q_{2}=n\left(u_{n}^{2}+1\right) .
$$

However, the new method yields $Q_{1}, Q_{2}$ and a third independent characteristic:

$$
Q_{3}=\left(u_{n}^{2}+1\right) \tan ^{-1}\left(u_{n}\right) .
$$

The ansatz $Q=Q\left(n, u_{n}\right)$ yields only a few independent characteristics for secondorder $\mathrm{O} \Delta \mathrm{Es}$. For instance, every linear homogeneous $\mathrm{O} \Delta \mathrm{E}$,

$$
u_{n+2}=p(n) u_{n+1}+q(n) u_{n},
$$


has precisely three such characteristics, namely

$$
Q_{1}=u_{n}, \quad Q_{2}=U_{1}(n), \quad Q_{3}=U_{2}(n),
$$

where $u_{n}=U_{1}(n)$ and $u_{n}=U_{2}(n)$ are linearly independent solutions of (3.13). Consequently, every $\mathrm{O} \Delta \mathrm{E}$ that is linearizable by a point transformation

$$
T:\left(n, u_{n}\right) \mapsto\left(n, \tilde{u}_{n}\left(n, u_{n}\right)\right)
$$

also has three characteristics of the form $Q=Q\left(n, u_{n}\right)$. By contrast, every secondorder ODE that is linear or linearizable by a point transformation has an eightparameter Lie algebra of point symmetry generators, which is isomorphic to $\mathfrak{H}((3)$. However, for linear $\mathrm{O} \Delta \mathrm{Es}$, the 'missing' characteristics are all of the form of (3.11).

Theorem 3.1. Every second-order linear homogeneous ordinary difference equation has an eight-dimensional Lie algebra of symmetry generators whose characteristics are linear in $u_{n+1}$. This Lie algebra is isomorphic to $\mathfrak{g l}(3)$.

Proof. A given linear homogeneous $\mathrm{O} \Delta \mathrm{E}$ (3.13), with two linearly independent solutions, $u_{n}=U_{1}(n)$ and $u_{n}=U_{2}(n)$, has two functionally independent first integrals that are linear in $u_{n}$ and $u_{n+1}$ :

$$
\phi^{1}\left(n, u_{n}, u_{n+1}\right)=\frac{u_{n} \mathcal{S} U_{2}-U_{2} u_{n+1}}{U_{1} \mathcal{S} U_{2}-U_{2} \mathcal{S} U_{1}}, \quad \phi^{2}\left(n, u_{n}, u_{n+1}\right)=\frac{U_{1} u_{n+1}-u_{n} \mathcal{S} U_{1}}{U_{1} \mathcal{S} U_{2}-U_{2} \mathcal{S} U_{1}} .
$$

From (2.20), every symmetry generator

$$
X=Q \frac{\partial}{\partial u_{n}}+\mathcal{S} Q \frac{\partial}{\partial u_{n+1}}
$$

can be rewritten in the form

$$
X=F^{1}\left(\phi^{1}, \phi^{2}\right) \frac{\partial}{\partial \phi^{1}}+F^{2}\left(\phi^{1}, \phi^{2}\right) \frac{\partial}{\partial \phi^{2}},
$$

where, for the first integrals (3.16),

$$
F^{i}\left(\phi^{1}, \phi^{2}\right)=X\left(\phi^{i}\left(n, u_{n}, u_{n+1}\right)\right)=\phi^{i}(n, Q, \mathcal{S} Q) .
$$

In particular, setting $Q=U_{j}(n)$ gives

$$
X=\frac{\partial}{\partial \phi^{j}} .
$$

Therefore, every one-parameter Lie group of symmetries of (3.13) has a characteristic of the form

$$
Q\left(n, u_{n}, u_{n+1}\right)=F^{1}\left(\phi^{1}, \phi^{2}\right) U_{1}(n)+F^{2}\left(\phi^{1}, \phi^{2}\right) U_{2}(n) .
$$

To find all characteristics that are linear in $u_{n+1}$, differentiate (3.18) twice with respect to $u_{n+1}$ and (using the fact that $U_{1}(n)$ and $U_{2}(n)$ are independent) obtain constraints on the functions $F^{i}$. A basis for the space of such characteristics is

$$
\begin{gathered}
Q_{1}=U_{1}(n), \quad Q_{2}=U_{2}(n), \quad Q_{3}=\phi^{1} U_{1}(n), \\
Q_{4}=\phi^{2} U_{1}(n), \quad Q_{5}=\phi^{1} U_{2}(n), \quad Q_{6}=\phi^{2} U_{2}(n), \\
Q_{7}=\left(\phi^{1}\right)^{2} U_{1}+\phi^{1} \phi^{2} U_{2}=\phi^{1} u_{n}, \quad Q_{8}=\phi^{1} \phi^{2} U_{1}+\left(\phi^{2}\right)^{2} U_{2}=\phi^{2} u_{n} .
\end{gathered}
$$

It is easy to check that the corresponding generators form a Lie algebra isomorphic to $\mathfrak{s l}(3)$. 
Note: it is not true that every $\mathrm{O} \Delta \mathrm{E}$ that is linearizable by a point transformation has an eight-dimensional Lie algebra whose characteristics are linear in $u_{n+1}$. For example, the $\mathrm{O} \Delta \mathrm{E}(3.1)$ can be linearized by the point transformation (3.15) with $\tilde{u}_{n}=1 / u_{n}$. However, there are no characteristics that are linear in $u_{n+1}$ other than those that we found earlier, which are independent of $u_{n+1}$. Byrnes et al. (1995) have derived necessary and sufficient conditions for a difference equation to be linearizable.

Theorem 3.1 generalizes a result of Levi et al. (1997), who showed that

$$
u_{n+2}=2 u_{n+1}-u_{n}
$$

has a Lie algebra that is isomorphic to $\mathfrak{g l}(3)$.

Just as for ODEs, it is usually not easy to find more than one characteristic of a given second-order linear homogeneous $\mathrm{O} \Delta \mathrm{E}$, namely

$$
Q=u_{n}=Q_{3}+Q_{6} .
$$

To obtain any other characteristic, one must find at least one solution of the $\mathrm{O} \Delta \mathrm{E}$ (or its adjoint). This severely limits the usefulness of symmetry methods for linear equations. For nonlinear $\mathrm{O} \Delta \mathrm{Es}$, however, symmetries of a given form can usually be found without too much difficulty, if they exist. The main problem is to choose a useful restriction on $Q$. For example, consider the $\mathrm{O} \Delta \mathrm{E}$

$$
u_{n+2}=-u_{n+1}-u_{n}+\frac{n}{u_{n+1}},
$$

which is a discrete Painlevé I equation (see Its et al. 1990). The LSC is

$$
Q(n+2, \omega, \mathcal{S} \omega)+\left(1+\frac{n}{u_{n+1}^{2}}\right) Q\left(n+1, u_{n+1}, \omega\right)+Q\left(n, u_{n}, u_{n+1}\right)=0
$$

where $\omega$ is the right-hand side of (3.19). It turns out that (3.20) has no solutions for which $Q\left(n, u_{n}, u_{n+1}\right)$ is linear in $u_{n+1}$, just as the continuous Painlevé ODEs have no Lie point symmetries.

For simplicity, we have concentrated on second-order O $\Delta$ Es. However, the same method can also be used to obtain symmetries of higher-order O $\Delta$ Es. If one uses a more general ansatz, such as $Q=Q\left(n, u_{n}, u_{n+1}\right)$, the method leads to a system of partial differential equations for $Q$. So far, we have chosen to eliminate $\mathcal{S}^{k} Q, k \geqslant 1$, to obtain a system that involves only $Q$ and its derivatives. This is not always the best strategy; sometimes it is better to obtain a system for $\mathcal{S}^{k_{0}} Q$ for some $k_{0}>0$. For example,

$$
u_{n+4}=\frac{u_{n+1}^{2}}{u_{n}}+u_{n}
$$

has only one characteristic of the form $Q=Q\left(n, u_{n}, u_{n+1}\right)$, namely $Q=c^{1} u_{n}$. This result is easy to obtain if differential elimination is used to derive a system for $S^{4} Q$, whereas the system for $Q$ appears intractable. This demonstrates that some experimentation may be needed if the standard reduction in favour of $Q$ leads to a system that is too hard to solve.

\section{How to use symmetries of $\mathrm{O} \Delta \mathrm{Es}$}

Remarkably, it seems that almost any symmetry method for ODEs has a counterpart for $\mathrm{O} \Delta \mathrm{Es}$. Usually only slight modification is needed to obtain the $\mathrm{O} \Delta \mathrm{E}$ methods. 
Here we use second-order $\mathrm{O} \Delta$ Es to demonstrate various methods. The generalization to higher-order problems is straightforward.

Given a symmetry generator for a second-order $\mathrm{O} \Delta \mathrm{E}$,

$$
X=Q \frac{\partial}{\partial u_{n}}+\mathcal{S} Q \frac{\partial}{\partial u_{n+1}},
$$

there exists an invariant,

$$
v_{n}=v\left(n, u_{n}, u_{n+1}\right),
$$

satisfying

$$
X v_{n}=0, \quad \frac{\partial v_{n}}{\partial u_{n+1}} \neq 0 .
$$

This invariant is found by the method of characteristics; it is a first integral of

$$
\frac{\mathrm{d} u_{n}}{Q}=\frac{\mathrm{d} u_{n+1}}{\mathcal{S} Q} .
$$

Moreover, every invariant function of $n, u_{n}$, and $u_{n+1}$ is a function of $n$ and $v_{n}$ only. For later use, we shall suppose that (4.2) can be inverted to obtain

$$
u_{n+1}=w\left(n, u_{n}, v_{n}\right)
$$

for some function $w$.

From (2.21),

$$
X\left(\mathcal{S} v_{n}\right)=\mathcal{S}\left(X v_{n}\right)=0,
$$

so $\mathcal{S} v_{n}$ is invariant: it is a function of $n$ and $v_{n}$ only. Thus, the solutions of

$$
u_{n+2}=\omega\left(n, u_{n}, u_{n+1}\right)
$$

satisfy a first-order $\mathrm{O} \Delta \mathrm{E}$ of the form

$$
v_{n+1}=\mathcal{S} v_{n}=\Omega\left(n, v_{n}\right) .
$$

If (4.6) can be solved (perhaps by exploiting further symmetries of (4.5), see below) then the general solution,

$$
v_{n}=v\left(n, u_{n}, u_{n+1}\right)=f\left(n ; c^{1}\right),
$$

is equivalent to the first-order $\mathrm{O} \Delta \mathrm{E}$

$$
u_{n+1}=w\left(n, u_{n}, f\left(n ; c^{1}\right)\right),
$$

which admits the symmetries generated by $X$. To solve (4.8), we need to obtain a canonical coordinate,

$$
s_{n}=s\left(n, u_{n}\right),
$$

that satisfies

$$
X s_{n}=1 .
$$


The most obvious choice of canonical coordinate is (see Joshi \& Vassiliou 1995)

$$
s\left(n, u_{n}\right)=\int \frac{\mathrm{d} u_{n}}{Q\left(n, u_{n}, w\left(n, u_{n}, f\left(n ; c^{1}\right)\right)\right)} .
$$

Note that

$$
X s_{n+1}=X\left(\mathcal{S} s_{n}\right)=\mathcal{S}\left(X s_{n}\right)=\mathcal{S}(1)=1=X s_{n},
$$

so $s_{n+1}-s_{n}$ is an invariant. Consequently,

$$
s_{n+1}=s_{n}+g\left(n, v_{n}\right)
$$

for some function $g$, and therefore (4.8) is equivalent to

$$
s_{n+1}=s_{n}+g\left(n, f\left(n ; c^{1}\right)\right) .
$$

The general solution of (4.11) is

$$
s_{n}=c^{2}+\sum_{r=n_{0}}^{n-1} g\left(r, f\left(r ; c^{1}\right)\right),
$$

where $n_{0}$ is any convenient integer.

If an $\mathrm{O} \Delta \mathrm{E}$ has an $N$-dimensional solvable Lie (sub)algebra of symmetry generators, the solvable structure can be exploited in exactly the same way as for ODEs. Consider the nonlinear $\mathrm{O} \Delta \mathrm{E}$

$$
u_{n+2}=\frac{2 u_{n+1}^{3}}{u_{n}^{2}}-u_{n+1} .
$$

The set of characteristics that are linear in $u_{n+1}$ is spanned by

$$
Q_{1}=\frac{u_{n+1}}{u_{n}}, \quad Q_{2}=u_{n} .
$$

The commutator $\left[X_{1}, X_{2}\right]$ has the characteristic

$$
\left[Q_{1}, Q_{2}\right] \equiv X_{1} Q_{2}-X_{2} Q_{1}=\frac{u_{n+1}}{u_{n}}=Q_{1}
$$

Therefore, the generators $X_{1}, X_{2}$ form a basis for a (transitive) non-abelian solvable Lie algebra, whose derived subalgebra is spanned by $X_{1}$. Consequently, $X_{1}$ should be used for the first reduction of order, so that the reduced $\mathrm{O} \Delta \mathrm{E}$ inherits the symmetries generated by $X_{2}$. The invariant $v_{n}$ of the group generated by $X_{1}$ satisfies

$$
X_{1} v_{n}=\left(\frac{u_{n+1}}{u_{n}} \frac{\partial}{\partial u_{n}}+\left\{\frac{2 u_{n+1}^{2}}{u_{n}^{2}}-1\right\} \frac{\partial}{\partial u_{n+1}}\right) v_{n}=0 .
$$

Using the method of characteristics, we obtain

$$
v_{n}=\frac{\left(u_{n+1}^{2}-u_{n}^{2}\right)}{u_{n}^{4}} .
$$

This reduces the $\mathrm{O} \Delta \mathrm{E}(4.13)$ to

$$
v_{n+1}=4 v_{n},
$$


which inherits the scaling symmetry generated by $X_{2}$. The general solution of (4.16) is

$$
v_{n}=c^{1} 4^{n}
$$

which is equivalent to

$$
u_{n+1}= \pm u_{n} \sqrt{1+c^{1} 4^{n} u_{n}^{2}} .
$$

However, the negative root is inconsistent with (4.13). Therefore, the canonical coordinate is

$$
s_{n}=\int \frac{\mathrm{d} u_{n}}{\sqrt{1+c^{1} 4^{n} u_{n}^{2}}}=\frac{1}{\sqrt{c^{1}} 2^{n}} \sinh ^{-1}\left(\sqrt{c^{1}} 2^{n} u_{n}\right) .
$$

Then (4.18) is equivalent to

$$
s_{n+1}=s_{n},
$$

whose general solution is $s_{n}=c^{2}$. In the original variables, the general solution of (4.13) is

$$
u_{n}=\frac{1}{\sqrt{c^{1}} 2^{n}} \sinh \left(c^{2} \sqrt{c^{1}} 2^{n}\right) .
$$

The above technique fails if $X_{2}$ is a scalar multiple of $X_{1}$, in which case the symmetry group generated by $X_{1}$ and $X_{2}$ is intransitive. Then, if $v_{n}$ satisfies $X_{1} v_{n}=$ 0 , it also satisfies $X_{2} v_{n}=0$. A single reduction of order can be achieved, but the remaining one-parameter Lie group acts trivially on the reduced $\mathrm{O} \Delta \mathrm{E}$ and cannot be used to solve it.

Intransitive two-dimensional Lie subgroups of point symmetries also occur for some second-order ODEs. They are of little consequence, because there is always a transitive two-dimensional subgroup of point symmetries as well (see Stephani 1989). However, for second-order O $\Delta \mathrm{Es}$, the usual ansätze may not yield a transitive group. The group generated by $X_{1}$ and $X_{2}$ is intransitive if

$$
\frac{Q_{2}}{Q_{1}}=\frac{\mathcal{S} Q_{2}}{\mathcal{S} Q_{1}}=\mathcal{S}\left(\frac{Q_{2}}{Q_{1}}\right)
$$

that is, if the ratio of the characteristics is a first integral:

$$
Q_{2} / Q_{1}=\phi \text {. }
$$

We now show how to construct another (functionally independent) first integral. The method depends upon whether or not $X_{1}$ and $X_{2}$ commute.

For now, it is most convenient to write the generators in terms of first integrals, with $X_{1}$ in normal form. Thus

$$
X_{1}=\frac{\partial}{\partial \phi^{1}}, \quad X_{2}=\phi \frac{\partial}{\partial \phi^{1}},
$$

for some first integral $\phi^{1}$, and there is an independent first integral, $\phi^{2}$, that is mapped to itself by the group action. From (4.21), we obtain

$$
\left[X_{1}, X_{2}\right]=\frac{\partial \phi}{\partial \phi^{1}} X_{1}
$$


Hence, if $\left[X_{1}, X_{2}\right] \neq 0$, then $\phi$ depends non-trivially on $\phi^{1}$. Now construct an invariant $v_{n}$ of $X_{1}$ as described earlier, so that the $\mathrm{O} \Delta \mathrm{E}$ amounts to

$$
v_{n+1}=\Omega\left(n, v_{n}\right) \text {. }
$$

Clearly, $v_{n}$ is a function of $n$ and $\phi^{2}$ only, so

$$
\phi^{2}=G\left(n, v_{n}\right)
$$

for some function $G$. To obtain $\phi^{2}$, we must find a solution of the functional difference equation

$$
G\left(n+1, \Omega\left(n, v_{n}\right)\right)=G\left(n, v_{n}\right) .
$$

(In practice, this is often quite easy.) Then $\phi$ and $\phi^{2}$ are functionally independent first integrals.

If $X_{1}$ and $X_{2}$ commute, then $\phi$ is a function of $\phi^{2}$ only. Indeed, without loss of generality, we can set

$$
\phi^{2}=\phi
$$

To obtain $\phi^{1}$, first note that

$$
X_{1} \phi^{1}=1
$$

and so $\phi^{1}$ is a canonical coordinate. Therefore,

$$
\phi^{1}=\int \frac{\mathrm{d} u_{n}}{Q_{1}\left(n, u_{n}, u_{n+1}\left(n, u_{n}, \phi^{2}\right)\right)}+G\left(n, \phi^{2}\right),
$$

for some function $G$. To obtain $G$ (up to an arbitrary function of $\phi^{2}$ ), we apply the condition

$$
\mathcal{S} \phi^{1}-\phi^{1}=0
$$

and solve the resulting first-order linear $\mathrm{O} \Delta \mathrm{E}$ using the standard method.

To illustrate this technique, consider the $\mathrm{O} \Delta \mathrm{E}$

$$
u_{n+2}=\frac{u_{n+1}^{2}}{u_{n}}+u_{n+1} .
$$

The symmetry generators whose characteristics are linear in $u_{n+1}$ form an abelian Lie algebra; the characteristics are linear combinations of

$$
Q_{1}=u_{n}, \quad Q_{2}=u_{n+1}-n u_{n} .
$$

It is easy to verify that

$$
\mathcal{S}\left(\frac{Q_{2}}{Q_{1}}\right)=\frac{Q_{2}}{Q_{1}},
$$

and, because the generators commute, we choose

$$
\phi^{2}=\frac{Q_{2}}{Q_{1}}=\frac{u_{n+1}}{u_{n}}-n .
$$

From (4.22),

$$
\phi^{1}=\ln \left|u_{n}\right|+G\left(n, \phi^{2}\right)
$$

where

$$
G\left(n+1, \phi^{2}\right)-G\left(n, \phi^{2}\right)=\ln \left|u_{n}\right|-\ln \left|u_{n+1}\right|=-\ln \left|n+\phi^{2}\right| .
$$


The general solution of $(4.26)$ is

$$
G\left(n, \phi^{2}\right)=A\left(\phi^{2}\right)-\ln \left|\Gamma\left(n+\phi^{2}\right)\right|,
$$

where $\Gamma(z)$ is the Gamma function and $A$ is an arbitrary function. Without loss of generality, we can set $A\left(\phi^{2}\right)=0$ and replace $\phi^{1}$ by its exponential,

$$
\tilde{\phi}^{1}=\frac{u_{n}}{\Gamma\left(n+\phi^{2}\right)} .
$$

Therefore, the general solution of (4.23) is

$$
u_{n}=c^{1} \Gamma\left(n+c^{2}\right) \text {. }
$$

\section{Direct construction of first integrals}

A recent paper by Anco \& Bluman (1998) describes a method for obtaining first integrals of a given ODE directly, whether or not any Lie symmetries are known. A simplified version of this method is given in Hydon (2000).

It is also possible to construct first integrals of $\mathrm{O} \Delta \mathrm{Es}$ directly, even if no symmetries are known. The starting point for this approach is the equation

$$
\mathcal{S} \phi=\phi, \quad \frac{\partial \phi}{\partial u_{n+N-1}} \neq 0 .
$$

For second-order $\mathrm{O} \Delta \mathrm{Es},(5.1)$ amounts to

$$
\phi\left(n+1, u_{n+1}, \omega\left(n, u_{n}, u_{n+1}\right)\right)=\phi\left(n, u_{n}, u_{n+1}\right), \quad \frac{\partial \phi}{\partial u_{n+1}} \neq 0 .
$$

(For brevity, we shall consider only second-order problems; the generalization to higher-order $\mathrm{O} \Delta \mathrm{Es}$ is entirely straightforward.)

It is convenient to introduce the functions

$$
\begin{aligned}
& P_{1}\left(n, u_{n}, u_{n+1}\right)=\frac{\partial \phi}{\partial u_{n}}\left(n, u_{n}, u_{n+1}\right), \\
& P_{2}\left(n, u_{n}, u_{n+1}\right)=\frac{\partial \phi}{\partial u_{n+1}}\left(n, u_{n}, u_{n+1}\right) .
\end{aligned}
$$

By differentiating (5.2) with respect to $u_{n}$ and $u_{n+1}$ in turn, we obtain

$$
\begin{aligned}
& P_{1}=\frac{\partial \omega}{\partial u_{n}} \mathcal{S} P_{2}, \\
& P_{2}=\mathcal{S} P_{1}+\frac{\partial \omega}{\partial u_{n+1}} \mathcal{S} P_{2} .
\end{aligned}
$$

Therefore, $P_{2}$ satisfies the second-order linear functional equation

$$
\mathcal{S}\left(\frac{\partial \omega}{\partial u_{n}}\right) \mathcal{S}^{2} P_{2}+\frac{\partial \omega}{\partial u_{n+1}} \mathcal{S} P_{2}-P_{2}=0 .
$$

Just as for the LSC, we obtain solutions of (5.7) by first choosing an ansatz, then differentiating repeatedly to obtain a differential equation for $P_{2}$. Given a solution 
$P_{2}$ of (5.7), it is straightforward to construct $P_{1}$. At this stage, it is necessary to check that the integrability condition

$$
\frac{\partial P_{1}}{\partial u_{n+1}}=\frac{\partial P_{2}}{\partial u_{n}}
$$

is satisfied. (This is because some solutions of (5.7) are not derived from any first integral.) If (5.8) holds, then the first integral $\phi$ is of the form

$$
\phi=\int\left(P_{1} \mathrm{~d} u_{n}+P_{2} \mathrm{~d} u_{n+1}\right)+G(n)
$$

where $G(n)$ is determined (up to an arbitrary constant) by substituting (5.9) into (5.2) and solving the resulting first-order linear $\mathrm{O} \Delta \mathrm{E}$.

To illustrate the method, consider the $\mathrm{O} \Delta \mathrm{E}$

$$
u_{n+2}=\frac{n}{n+1} u_{n}+\frac{1}{u_{n+1}} .
$$

We use the ansatz $P_{2}=P_{2}\left(n, u_{n}\right)$; then (5.7) amounts to

$$
\frac{n+1}{n+2} P_{2}(n+2, \omega)-\frac{1}{u_{n+1}^{2}} P_{2}\left(n+1, u_{n+1}\right)-P_{2}\left(n, u_{n}\right)=0 .
$$

Using the symmetry-finding algorithm of $\S 3$, we obtain a single solution (up to an arbitrary constant factor):

$$
P_{2}=n u_{n}
$$

Therefore,

$$
P_{1}=n u_{n+1},
$$

and the integrability condition is satisfied. From (5.9),

$$
\phi=n u_{n} u_{n+1}+G(n)
$$

and, hence,

$$
\mathcal{S} \phi-\phi=G(n+1)-G(n)+n+1=0 .
$$

(Note: no matter how complicated the original $\mathrm{O} \Delta \mathrm{E}$ is, the function $G$ always satisfies a first-order linear $\mathrm{O} \Delta \mathrm{E}$ that is easily solved.) In this example,

$$
G(n)=-\frac{1}{2}(n(n+1))
$$

(up to an irrelevant constant). Therefore, we have obtained the first integral

$$
\phi=n u_{n} u_{n+1}-\frac{1}{2}(n(n+1)) .
$$

The general solution to this particular problem can be found by rewriting $\phi=c^{1}$ as a first-order linear $\mathrm{O} \Delta \mathrm{E}$ for $v_{n}=\ln \left|u_{n}\right|$ :

$$
v_{n+1}+v_{n}=\ln \left|\frac{1}{2}(n+1)+\left(c^{1} / n\right)\right| .
$$


By using the standard method for such $\mathrm{O} \Delta \mathrm{Es}$, we obtain the general solution,

$$
v_{n}=(-1)^{n}\left(c^{2}+\sum_{k=n_{0}}^{n}(-1)^{k} \ln \left|\frac{1}{2} k+\frac{c^{1}}{k-1}\right|\right)
$$

here $n_{0}$ is a suitably chosen integer.

Just as for symmetries, the need to restrict $P_{2}$ means that it is not always possible to find solutions of (5.7). Furthermore, for some O $\Delta \mathrm{Es}$, including the discrete Painlevé I equation (3.19), the functional equations for $P_{2}$ and $Q$ are identical. Then it is equally difficult to find symmetries and first integrals. This occurs when the $\mathrm{O} \Delta \mathrm{E}$ arises from a variational principle, which will be treated in a separate paper. Maeda (1980) discusses the Lagrangian and Hamiltonian frameworks for first-order systems of $\mathrm{O} \Delta \mathrm{Es}$.

To solve a second-order $\mathrm{O} \Delta \mathrm{E}$, one usually needs either two independent characteristics $Q$ or two functions $P_{2}$ that satisfy the integrability condition. However, any pair of functions $\left(P_{1}, P_{2}\right)$ satisfying $(5.5)$ and (5.6) can be combined with characteristics of Lie symmetries to obtain first integrals, as follows.

Theorem 5.1. Given a second-order ordinary difference equation (3.7), suppose that $\left(P_{1}, P_{2}\right)$ solves (5.5), (5.6), and that $Q$ is the characteristic of a one-parameter Lie group of symmetries. Then

$$
\phi=P_{1} Q+P_{2} \mathcal{S} Q
$$

is either a first integral or a constant.

Proof. We use the LSC to show that $\mathcal{S} \phi=\phi$, as follows:

$$
\begin{aligned}
\mathcal{S} \phi & =\left(\mathcal{S} P_{1}\right)(\mathcal{S} Q)+\left(\mathcal{S} P_{2}\right)\left(\mathcal{S}^{2} Q\right) \\
& =\left(\mathcal{S} P_{1}\right)(\mathcal{S} Q)+\left(\mathcal{S} P_{2}\right)\left(\frac{\partial \omega}{\partial u_{n+1}} \mathcal{S} Q+\frac{\partial \omega}{\partial u_{n}} Q\right) \\
& =P_{1} Q+P_{2} \mathcal{S} Q \\
& =\phi .
\end{aligned}
$$

Note that theorem 5.1 does not require the integrability condition (5.8) to hold. However, if (5.8) is satisfied, it may be possible to construct two functionally independent first integrals from one pair $\left(P_{1}, P_{2}\right)$ and one characteristic.

\section{Discussion and conclusions}

In this paper, we have developed new methods for finding and using symmetries of a given $\mathrm{O} \Delta \mathrm{E}$. Maeda $(1980,1987)$ was the first to derive a restricted version of the LSC for first-order $\mathrm{O} \Delta \mathrm{Es}$ (on the assumption that the characteristic is independent of $n$ ). Maeda pointed out the difficulty of solving the LSC, which is a functional equation. He gave some examples for which symmetries can be found by adopting a very limited ansatz. Since then, several different approaches have been proposed for solving the LSC for higher-order difference equations. We now compare these approaches with ours. 
Quispel \& Sahadevan (1993) used a Laurent series expansion about a fixed point at infinity to obtain point symmetries. This approach works if there is such a fixed point, but there is no guarantee that the user will be able to sum the series to obtain the symmetries in closed form. (This is necessary in order to calculate invariants and canonical coordinates.) Our method provides the symmetries in closed form, irrespective of the existence and location of fixed points. Furthermore, it enables the user to deal with symmetries other than point symmetries (with the aid of computer algebra, if necessary). Quispel \& Sahadevan (1993) imposed the additional restriction that symmetries should commute in order to obtain a double reduction of order. This is unnecessary; we have shown that Lie's method for second-order ODEs can be adapted to $\mathrm{O} \Delta \mathrm{Es}$ with a two-parameter symmetry group, whether or not the group is abelian or transitive. Quispel \& Sahadevan (1993) studied difference equations in which the independent variable $n$ is continuous. They pointed out that for equations in which $n$ is an integer, one need only replace the arbitrary unit-periodic functions in their symmetries by arbitrary constants. The converse is also true: our approach is generalized to equations with continuous $n$ by replacing arbitrary constants with arbitrary unit-periodic functions. Furthermore, if $n$ is continuous, our method can be extended to determine symmetries whose characteristic depends on derivatives of $u$ with respect to $n$. For example, every $\mathrm{O} \Delta \mathrm{E}$ that is autonomous (i.e. that does not explicitly involve $n$ ) is invariant under the one-parameter Lie group whose characteristic is

$$
Q=\frac{\mathrm{d} u_{n}}{\mathrm{~d} n} .
$$

(This group is equivalent to the group of translations in $n$.)

Levi et al. (1997) also consider difference equations for which the independent variable is continuous. They describe a slightly different approach for dealing with difference equations that arise as discretizations of differential equations with known symmetries. They expand the LSC as a series in powers of $u(n)$, and seek symmetries that are more general than point symmetries by allowing coefficients that are functions of $n$ and the shift operator. Various extra restrictions are imposed by requiring that the symmetries should be consistent with those of the discretized differential equation in the appropriate limit. Generally speaking, the expressions derived by Levi et al. (1997) are far more complicated than ours. For example, the $\mathrm{O} \Delta \mathrm{E}$

$$
u_{n+2}=2 u_{n+1}-u_{n}
$$

has the $\mathfrak{G l}(3)$ Lie algebra given in the proof of theorem 3.1, with

$$
U_{1}(n)=1, \quad U_{2}(n)=n .
$$

This result is contained in eqns (3.38) and (3.39) of Levi et al. (1997) in a more complicated form.

If an $\mathrm{O} \Delta \mathrm{E}$ arises as a discretization of an ODE, it will contain the step lengths as parameters. Sometimes it is advantageous to treat such parameters as separate variables, and to consider symmetries that act on the step lengths as well as on $u(n)$. This idea was suggested by Dorodnitsyn and co-workers (Dorodnitsyn 1994; Dorodnitsyn et al. 2000), who used it to construct discretizations having given symmetries. Conversely, to find out whether a given discretization has any such symmetries, we can adapt the method outlined in the current paper, as the following example shows. 
Levi et al. (1997) considered the $\mathrm{O} \Delta \mathrm{E}$

$$
u_{n+2}=2 u_{n+1}-u_{n}+\sigma^{2}\left(u_{n+1}\right)^{2},
$$

which is a centred-difference approximation, with uniform step length $\sigma$, to the ODE

$$
u^{\prime \prime}=u^{2} \text {. }
$$

The ODE is invariant under translations in the independent variable, $x$, and under scalings in $x$ and $u$. Levi et al. (1997) found a discrete analogue of the translation symmetries, by treating $n$ as a continuous variable. However, they were unable to obtain a discrete analogue of the scalings. The reason for this is apparent from the $\mathrm{O} \Delta \mathrm{E}$ : scalings in $x$ are replaced by scalings in $\sigma$. In fact, the scaling group can be found using our method with the ansatz

$$
X=Q\left(n, u_{n}\right) \frac{\partial}{\partial u_{n}}+q(\sigma) \frac{\partial}{\partial \sigma} .
$$

(More generally, $Q$ may depend upon all parameters.) The scaling symmetries are generated by

$$
X=2 u_{n} \frac{\partial}{\partial u_{n}}-\sigma \frac{\partial}{\partial \sigma} .
$$

The chief difficulty in finding symmetries of $\mathrm{O} \Delta \mathrm{Es}$ lies in choosing appropriate restrictions on $Q$. In this paper, we have looked mainly at second-order examples, choosing $Q\left(n, u_{n}, u_{n+1}\right)$ to be linear in $u_{n+1}$. Our method also works for higher-order $\mathrm{O} \Delta \mathrm{Es}$, and for other restrictions; we only need each unknown function to be invariant under a first-order differential operator. Nevertheless, just as for ODEs, it is often not possible to obtain symmetries using a simple restriction.

For brevity, we have restricted our discussion to O $\Delta$ Es. However, much work has been done on partial difference equations (see Floreanini \& Vinet 1995; Levi et al. 1997), and on differential-difference equations (see Quispel et al. 1992; Levi \& Winternitz 1993, 1996). For partial difference equations, the LSC is a functional equation. A modified version of our method can be used to find symmetries, as will be described in a separate paper. (The chief obstacle to obtaining symmetries is the complexity of the calculations in the differential elimination stage.) Having found Lie symmetries, they may be used in principle to obtain group-invariant reductions. However, one significant difficulty is that it is often not possible to solve the invariant surface condition, which is typically a quasilinear first-order partial difference equation. At present, there does not seem to be general agreement on what is the best way to determine group-invariant solutions.

Our method can also be adapted for use with differential-difference equations. However, much progress has already been made with such equations by equating powers of derivatives in the LSC, which is the same technique that is used to obtain Lie symmetries of differential equations. Therefore, it seems that our technique is most needed for dealing with symmetries of pure difference equations.

\section{References}

Anco, S. C. \& Bluman, G. 1998 Integrating factors and first integrals for ordinary differential equations. Eur. J. Appl. Math. 9, 245-259. 
Anderson, I. M., Kamran, N. \& Olver, P. J. 1993 Internal, external and generalized symmetries. Adv. Math. 100, 53-100.

Bluman, G. W. \& Kumei, S. 1989 Symmetries and differential equations. Springer.

Byrnes, G. B., Sahadevan, R. \& Quispel, G. R. W. 1995 Factorizable Lie symmetries and the linearization of difference equations. Nonlinearity 8, 443-459.

Dorodnitsyn, V. A. 1994 Symmetry of finite-difference equations. In CRC handbook of Lie group analysis of differential equations (ed. N. H. Ibragimov), vol. 1, pp. 365-403. Boca Raton, FL: CRC Press.

Dorodnitsyn, V. A., Kozlov, R. \& Winternitz, P. 2000 Lie group classification of second-order ordinary difference equations. J. Math. Phys. 41, 480-504.

Floreanini, R. \& Vinet, L. 1995 Lie symmetries of finite-difference equations. J. Math. Phys. 36, 7024-7042.

Gaeta, G. 1993 Lie-point symmetries of discrete versus continuous dynamical systems. Phys. Lett. A 178, 376-384.

Hydon, P. E. 2000 Symmetry methods for differential equations. Cambridge University Press.

Its, A. R., Kitaev, A. V. \& Fokas, A. S. 1990 The isomonodromy approach in the theory of 2-dimensional quantum gravitation. Russ. Math. Surv. 45, 155-156.

Joshi, N. \& Vassiliou, P. J. 1995 The existence of Lie symmetries for first-order analytic discrete dynamical systems. J. Math. Analysis Appl. 195, 872-887.

Levi, D. \& Winternitz, P. 1993 Symmetries and conditional symmetries of differential-difference equations. J. Math. Phys. 34, 3713-3730.

Levi, D. \& Winternitz, P. 1996 Symmetries of discrete dynamical systems. J. Math. Phys. 37, 5551-5576.

Levi, D., Vinet, L. \& Winternitz, P. 1997 Lie group formalism for difference equations. J. Phys. A 30, 633-649.

Maeda, S. 1980 Canonical structure and symmetries for discrete systems. Math. Jap. 4, 405-420.

Maeda, S. 1987 The similarity method for difference equations. IMA J. Appl. Math. 38, 129-134.

Olver, P. J. 1993 Applications of Lie groups to differential equations, 2nd edn. Springer.

Quispel, G. R. W. \& Sahadevan, R. 1993 Lie symmetries and the integration of difference equations. Phys. Lett. A 184, 64-70.

Quispel, G. R. W., Capel, H. W. \& Sahadevan, R. 1992 Continuous symmetries of differentialdifference equations: the Kac-van Moerbeke equation and Painlevé reduction. Phys. Lett. A 170, 379-383.

Stephani, H. 1989 Differential equations: their solution using symmetries. Cambridge University Press. 